\title{
Wearable Smart Spectacles using Arduino
}

\author{
Mr. Vishal Thorat ${ }^{1}$, Ms. Pranesha Naik ${ }^{2}$, Mr. Pranav Shetty ${ }^{3}$, Mr. Manish Singh ${ }^{4}$ \\ Guided By: Kishor G Sawarkar ${ }^{5}$ \\ 1,2,3,4,5 Deparment of Electronics and Telecommunication Engineering, \\ Rajiv Gandhi Institute of Technology, \\ Mumbai, Maharashtra, India
}

\begin{abstract}
Smart spectacles or smart glasses are wearable smart glasses that add information alongside to what user sees. Alternatively, smart glasses are sometimes defined as wearable computer glasses that are able to change their optical properties at runtime. Smart sunglasses which are programmed to change tint by electronic means these are an Example of the latter type of smart glasses. Superimposing information onto a field of view is achieved through an optical head-mounted display (OHMD) or embedded wireless glasses with transparent heads-up display (HUD) or augmented reality (AR) overlay. These systems have the capability to reflect projected digital images as well as allow the user to see through it or see better with it. While early models can perform basic tasks, such as serving as a front end display for a remote system, as in the case of smart glasses utilizing cellular technology or Wi-Fi, modern smart glasses are effectively wearable computers which can run self-contained mobile apps. Some are hands free and can communicate with the Internet via natural language voice commands, while others use touch buttons. Like other computers, smart glasses may collect information from internal or external sensors. It may control or retrieve data from other instruments or computers. It may support wireless technologies like Bluetooth, Wi-Fi, and GPS. A small number of models run a mobile operating system and function as portable media players to send audio and video files to the user via a Bluetooth or Wi-Fi headset. Some smart glasses models also feature full lifelogging and activity tracker capability. Smart glasses devices may also have features found on a smartphone
\end{abstract}

Key Words: Wearable smartglass, Arduino based notification glass, Arduino based Bluetooth smart glass, Smart spectacles, Smart spectacles using Arduino.

\section{INTRODUCTION}

As technology is growing rapidly and integrating itself to all aspects of people's life, designers and developers tried to provide a more pleasant experience of technology to people. One of the technology trends which aim to make life easier is wearable computing. Wearable's aim to assist people to be in control of their life by augmenting the real life with extra information constantly and ubiquitously. One of the growing trends of wearable computing is Head Mounted Displays (HMD), as the head is a great gateway to receive audio, visual and hectic information. A useful technique for all kinds of people including handicapped/disabled. In this project, we will make a wearable extension, and it will be used to send notifications of calls and messages received on mobile phones, and also show time and date, all in front of wearer's eye. Smart- Glasses are the wearable computing device used as an extension, which can be attached to the spectacles or sunglasses of the wearer, and can be paired with Smart Phones, via Bluetooth. This extension, contains an Arduino Microcontroller having ATmega328p microprocessor, which is programmed to connect with Smart-Phones through a Smart- phone application. A Bluetooth module, named HC-05 is interfaced with ATmega328p, which is used to connect with smart-phones. A battery / Re-chargeable battery of $5 \mathrm{~V}$ is used as power supply for Smart-Glass. An SSD1306, 0.96" OLED display is interfaced with ATmega328p, which is used to display the data received from Smart-phones. Smart-Phone application is used to transmit data of the phone, i.e. Date, Time, Notifications of Phone call and Text messages.

\subsection{Related Work}

\section{A) MIT App Inventor}

MIT App Inventor is a web application integrate development environment originally provided by Google, and now maintained by the Massachusetts Institute of Technology (MIT). It allows newcomers to computer programming to create application software apps for two operating systems (OS) Android operating system, Android, and iOS. It uses a graphical user interface (GUI) very similar to the programming languages Scratch programming language and the Star Logo, which allows users to drag and drop visual objects to create an application that can run on mobile devices. In creating App Inventor, Google drew upon significant prior research in educational computing, and work done within Google on online development environments.

Considering our system it has to work with the Bluetooth module to access the notifications of a smart phone to be displayed on the screen. So in order to work building an android application was the first concern, considering different modules network buttons to be accessed and placed accordingly. Certain blocks in app inventor that interacts with the Bluetooth client are considered and build according to the requirements so pass on the notifications of the smart phone to the display unit connected to the spectacles.

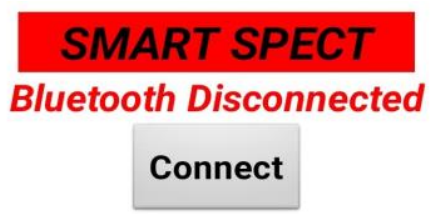

$$
\begin{gathered}
22: 3: 2020 \\
14: 45: 43
\end{gathered}
$$

85

Vodafone INLTE

Error 515: Not connected to a Bluetooth device.

Fig 1: Mobile application UI 


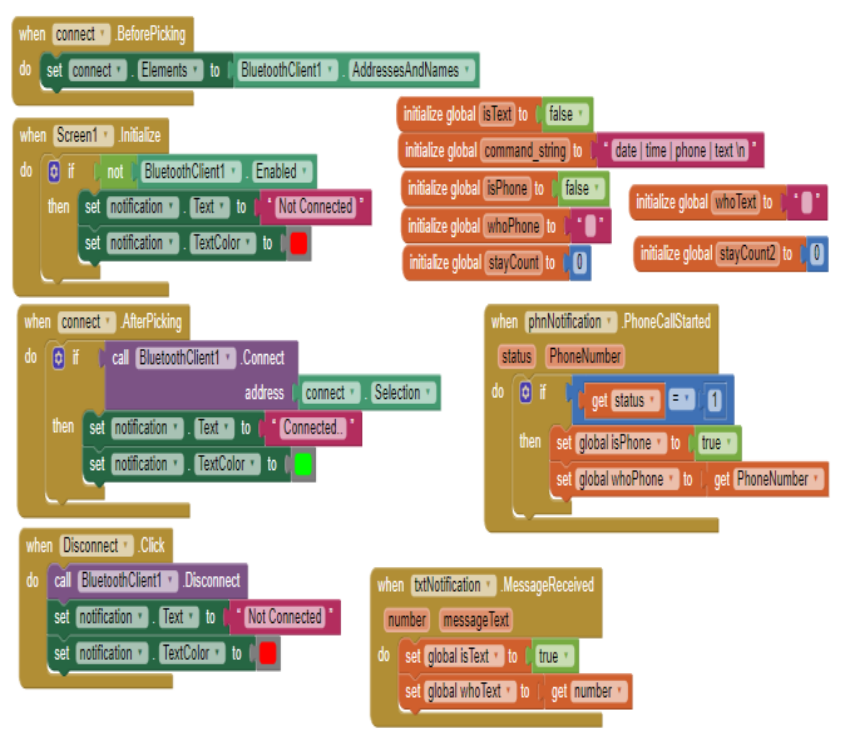

Fig 2: Application Structure Blocks

App Inventor programs describe how the phone should respond to certain events: a button has been pressed, the phone is being shaked, the user is dragging her finger over a canvas, etc. Some commands require one or more input values (also known as parameters or arguments) to completely specify their action. So specifically certain blocks have been created to access the Bluetooth client and pass on the notifications to the display unit for optimum working of the system.

\section{PROPOSED SYSTEM}

The smart glass module works on the principle of reflection and focusing of light. The information displayed on the OLED screen will be shown on the anti-reflecting glass by reflecting it with the help of mirror and then it is focused on the screen with the help of a focal lens. The module is powered by $280 \mathrm{~mA}$ Lithium Polymer battery which can be charged with the help of USB charger circuit and the power to the Arduino Nano is controlled with the help of a switch. The Bluetooth HC-05 module is controlled by Arduino Nano for displaying the received output on the OLED display. The Arduino pro Nano acts as CPU in this module it is interfaced with the Bluetooth module, OLED display.

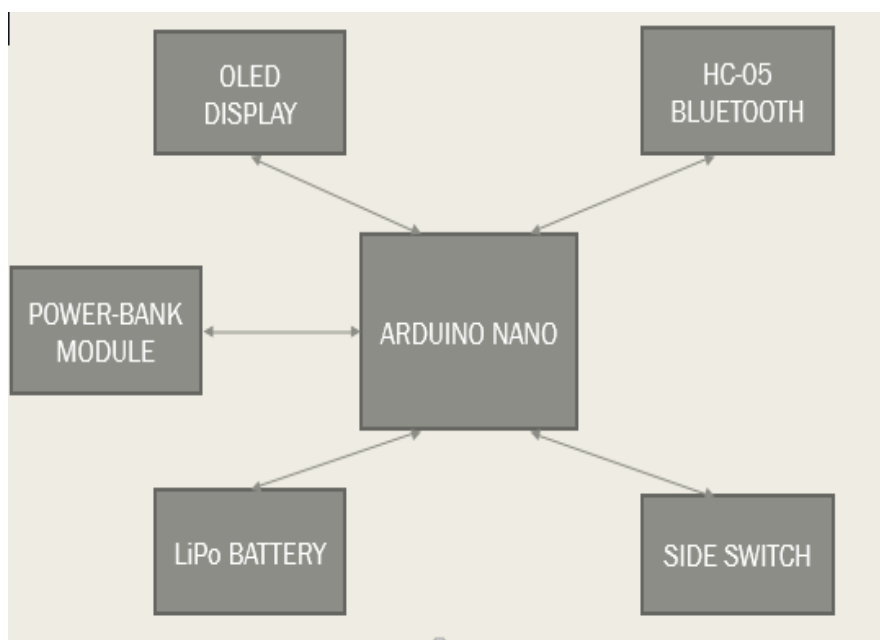

Fig 3: Block diagram of system
An Arduino Micro-controller having ATmega328p microprocessor, which is programmed to connect with SmartPhones through a Smart-phone application. A Bluetooth module, named HC-05 is interfaced with ATmega328p, which is used to connect with smart-phones. A battery / Re-chargeable battery of $5 \mathrm{~V}$ is used as power supply for Smart-Glass. An SSD1306, 0.96" OLED display is interfaced with ATmega328p, which is used to display the data received from Smart-phones. Smart-Phone application is used to transmit data of the phone, i.e. Date, Time, Notifications of Phone call and Text messages.

The following are the main steps that are implemented during the whole process:

1. Notifications Received

2. Encoding.

3. Transmitting and Receiving.

4. Decode and Process.

5. Execution

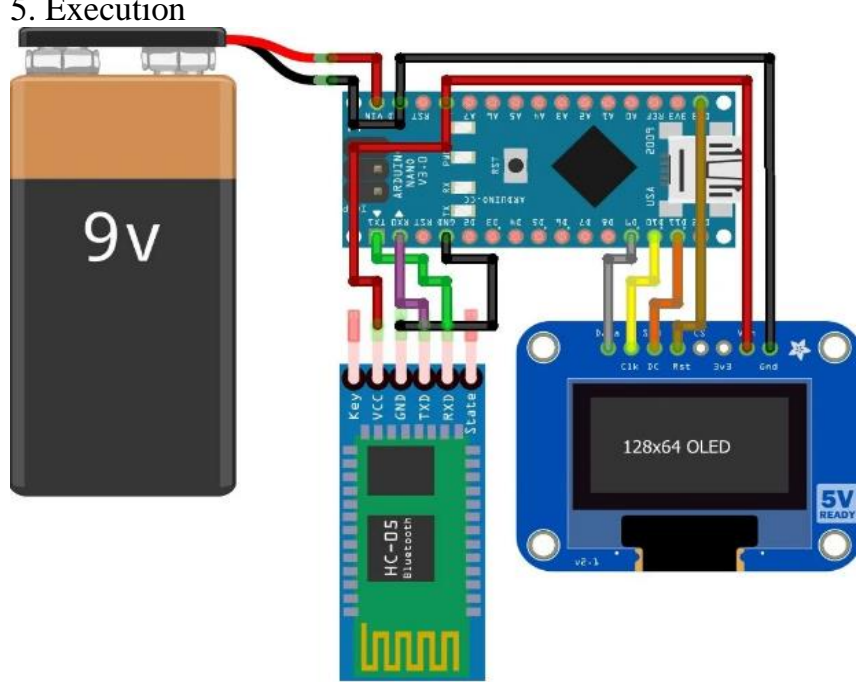

Fig 4: Circuit connections

The above circuit will interact with the application through the Bluetooth module in the following manner

1. It will receive the notifications on the smart phone

2. This notification will be encoded via the mobile application to be sent via the Bluetooth client

3. The encoded signal data will be received by the Bluetooth module $\mathrm{HC} 05$ and passes onto the Arduino Nano for decoding and forwarding.

4. The Arduino will forward the signal to the display unit and will receive and optimum output.

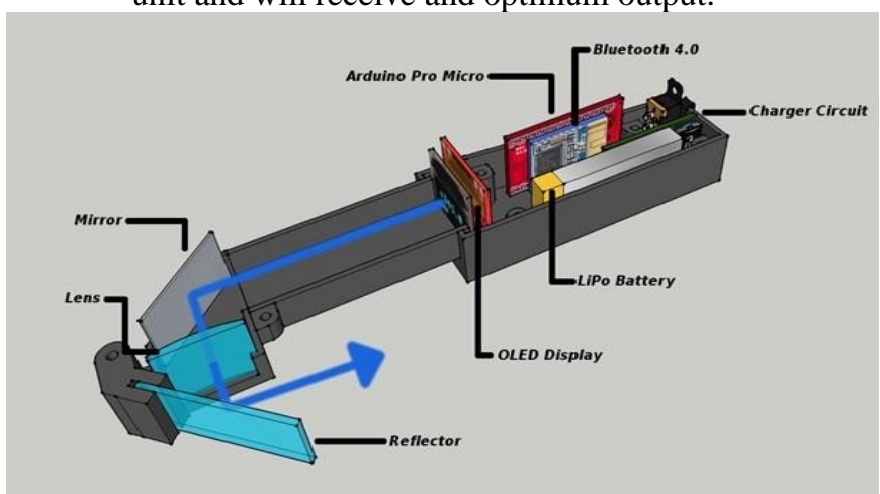

Fig 5: Final design of the system 


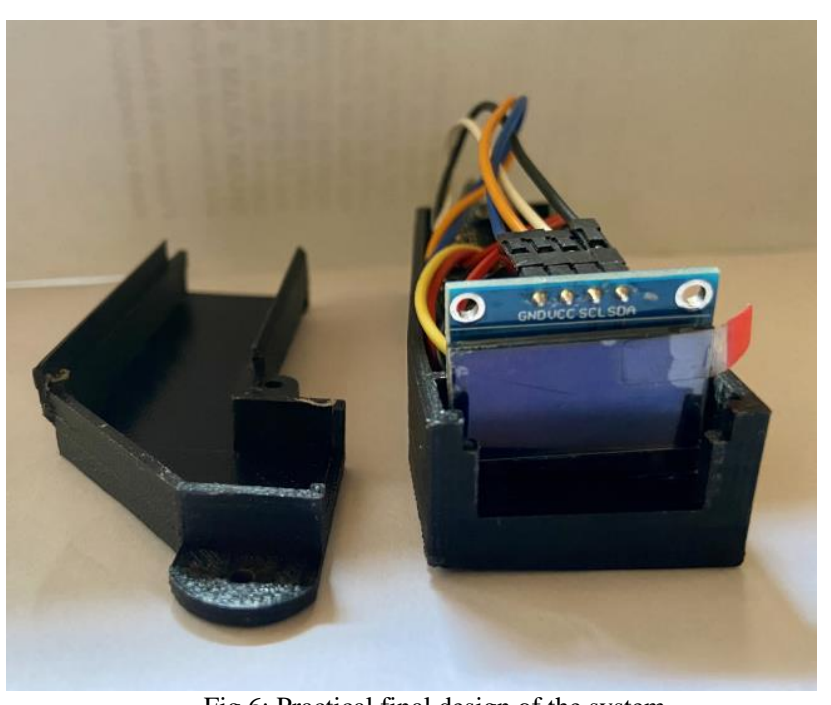

Fig 6: Practical final design of the system

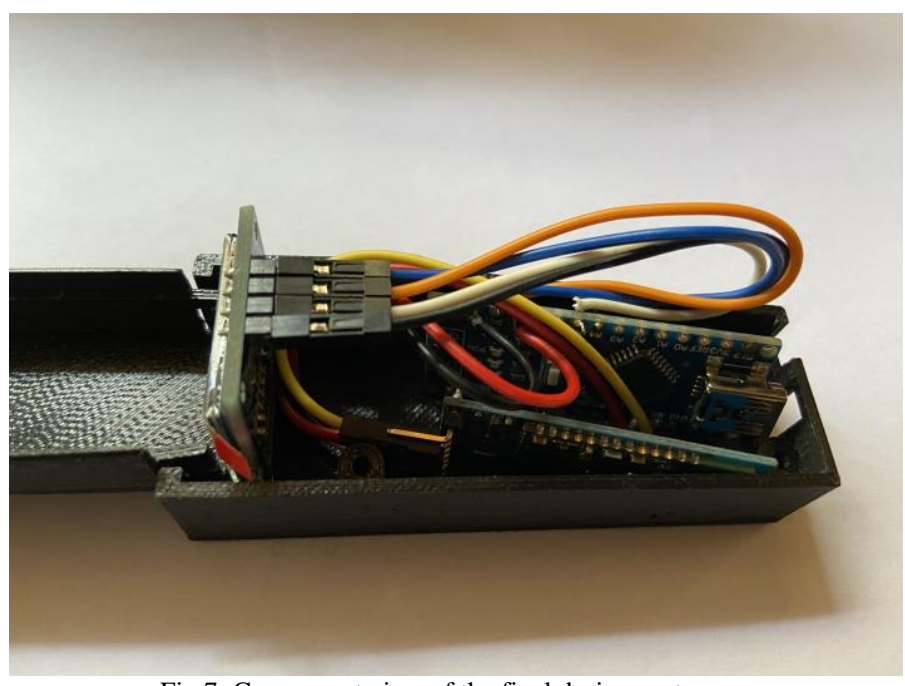

Fig 7: Component view of the final design system

\section{RESULTS}

\section{Experimental results}

As the system provides basic notifications which are accessed through the smart phone connected via Bluetooth HC05.

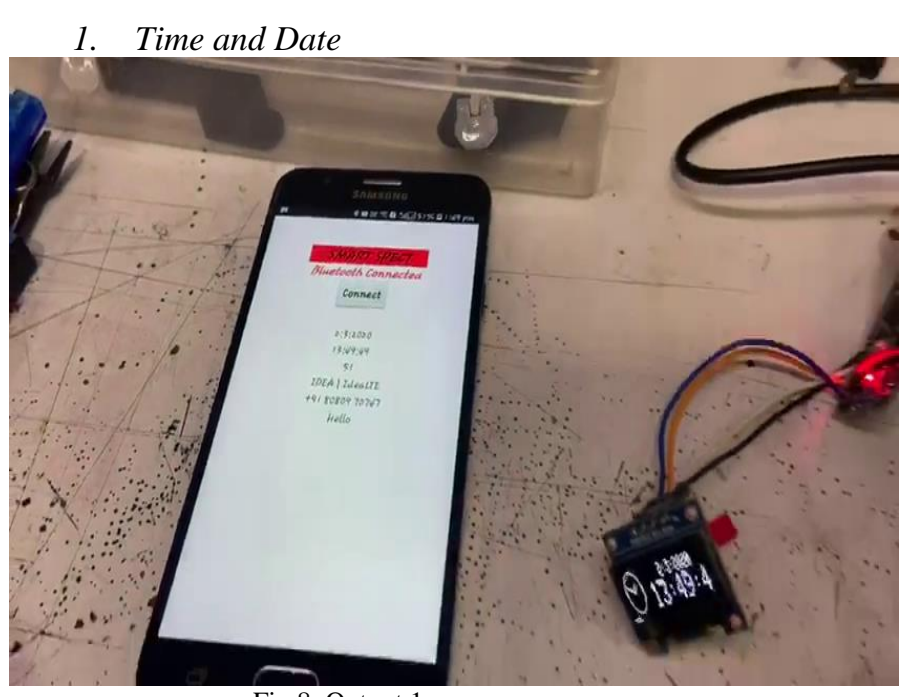

Fig 8: Output 1
We are able to detect the local time on the system exactly synchronized with the smart phone.

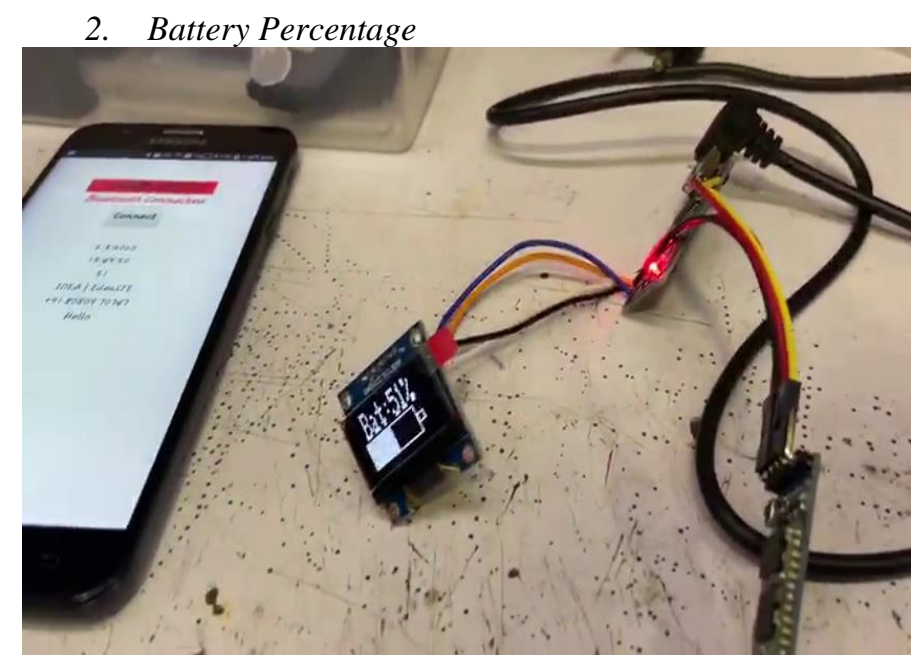

Fig 9: Output 2

Real time battery percentage is been noted and it gets updated every time the cycle repeats.

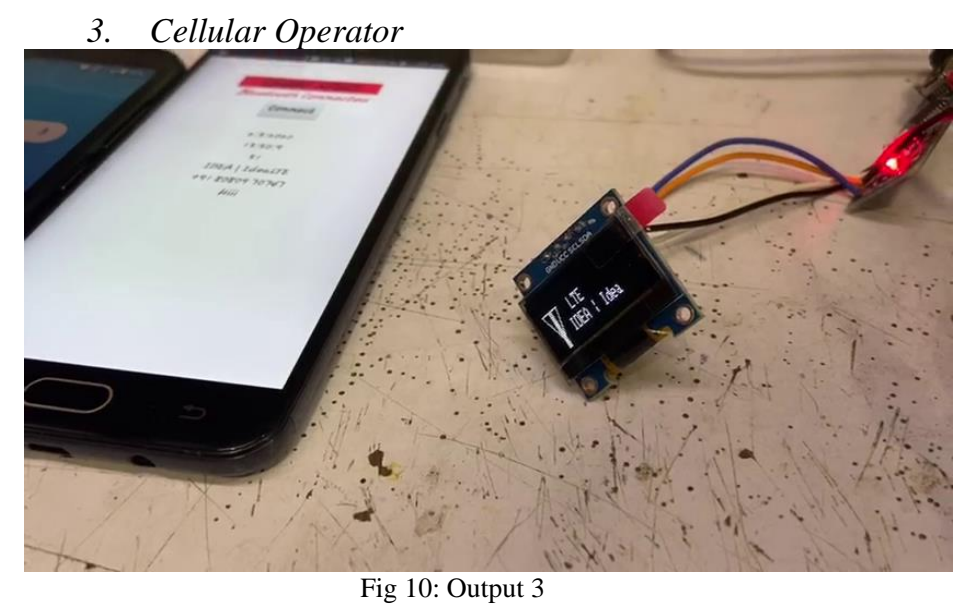

The Smart phone having a primary SIM is been detected by the system and displayed accordingly

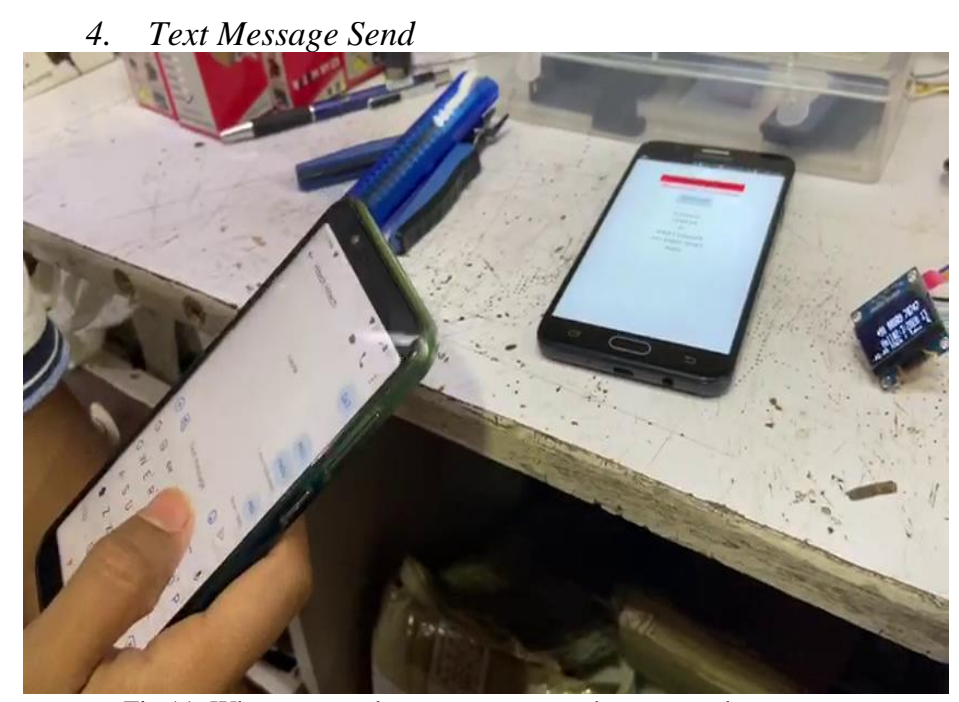

Fig 11: When one send a text message to the system phone 
The text message is sent from an unknown device to the system phone and it reflects in showing the message delivered to it with the phone number as well as the message itself.

\section{Text Message Receive}

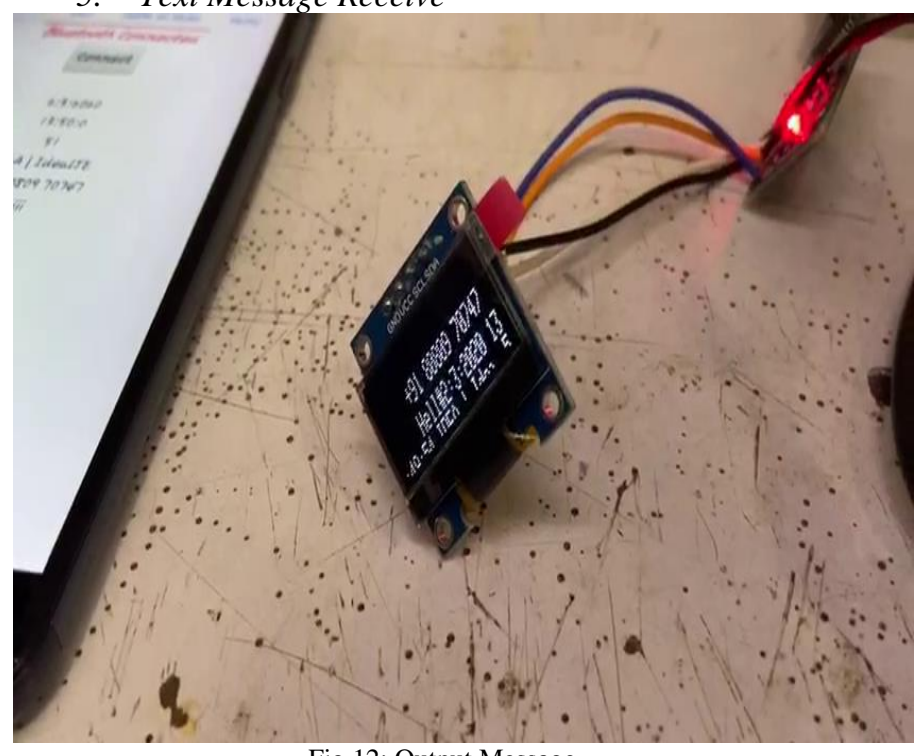

Fig 12: Output Message

Here the message received by the phone is displayed in the form of notifications with unknown number being displayed as well as the message that is been delivered.

\section{CONCLUSIONS}

The system provides satisfactory results the system provides basic notifications to the display unit such as SMS, time, date and information about the sim card the phone is using in a continuous loop. This system is placed in a $3 \mathrm{~d}$ case which fits with any spectacles and can be used as a portable device.

After which one is able to observe the date, time, cellular operator, the text message.

\section{FUTURE SCOPE}

According to the results we have obtained the system tend to take time for updating the new notifications easily that is the efficiency can be increased. The text message received can uphold only 5 words at one output in the display screen, so longer than that is not able to be received by the system. Calling scope can be added to this system as general OS permissions are not been able to get access through our system to give the calling notification facility.

\section{REFERENCES}

[1] M Alain Mauler - Arduino Data Glasses 2016

[2] Massimo Banzi and Michael Shiloh - Getting Started With Arduino - 3rd edition by Maker India Publication.

[3] Brian L. Due - The future of smart glasses: An essay about challenges and possibilities with smart glasses 2014

[4] Rufat Rzayev1, Paweł W. Wo'zniak1- Reading on Smart Glasses: The Effect of Text Position.

[5] Wearable Computing: A First Step Toward Personal Imaging, IEEE Computer, Vol. 30

\section{BIOGRAPHIES}

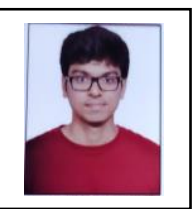

VISHAL THORAT

Currently pursuing under graduation final year in the branch of EXTC at Rajiv Gandhi Institute of

Technology, Versova.

Team Leader of the Project Group

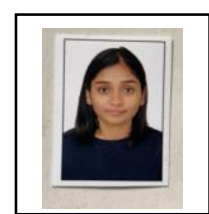

\section{PRANESHA NAIK}

Currently pursuing under graduation final year in the branch of EXTC at Rajiv Gandhi Institute of Technology, Versova.

Team Member of the Project Group

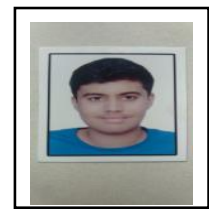

\section{PRANAV SHETTY}

Currently pursuing under graduation final year in the branch of EXTC at Rajiv Gandhi Institute of Technology, Versova.

Team Member of the Project Group

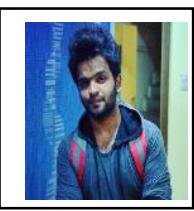

\section{MANISH SINGH}

Currently pursuing under graduation final year in the branch of EXTC at Rajiv Gandhi Institute of Technology, Versova.

Team Member of the Project Group

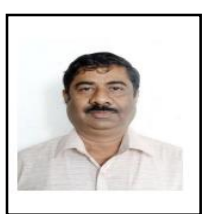

Prof. KISHOR G. SAWARKAR

Assistant Professor in Electronics \& Telecommunication Department. Project Guide at Rajiv Gandhi Institute of Technology, Versova. 